\title{
The Fractal Dimension Research of Chinese and American Beef Marbling Standards Images
}

\author{
Jianwen Chen, Meiying Liu*, and Li Zong \\ College of Engineering, Huazhong Agricultural University, Wuhan, \\ Hubei Province 430070
}

\begin{abstract}
Beef marbling level is the most important indicators in the evaluation of beef quality. The fractal dimension is closely related to marbling level. In this paper, the theory of fractal dimension is used to analyze beef marbling standards images in China and USA. After comparing several different dimension calculation method, the final method is improved box-counting dimension. Linear regression model of dimension calculation value with this method and marbling level is built. The model results are satisfactory through examination. For further use of samples, this model settles the foundation to establish the grade evaluation methods.
\end{abstract}

Keywords: marbling, longissimus dorsi, fractal dimension, beef quality, standards images.

\section{Introduction}

Assessment of beef quality, is highly valued in foreign countries. Developed countries proposed grading standards earlier, and generated significant economic benefits. China had a late start in this area, and began research in 2000. The assessment of beef quality, marbling quality is the most important indicator. Marbling usually refers to the section pattern of cattle at the 11-13 sternocostal or 5-7 sternocostal fat department [1] of the longissimus dorsi. Marbling is divided into six levels in USA, from 3 to 8 , the quality is getting better and better. Marbling level of 1 and 2 beefs are chopped to use in tin system, do not directly sell. In China, marbling is divided into four levels. From 1 to 4 , the quality is getting worse. Beef quality grading has a significant economic effect. Different quality grades of beefs have the difference prices. However, in China and abroad, the main method of beef grade assessment is manual measurement and artificial sensory evaluation, which have low efficiency and error shortcomings.

Fractal theory was proposed by Mandelbrot. The fractal dimension is important object in fractal theory research [2]. It is a number which describes the complexity of fractal collection. Recently the fractal theory is applied to the assessment of marbling in China and abroad. Some scholars expressed their views, and had mixed results.

\footnotetext{
* Supported by the fundamental research funds for the central universities, program No.52902090020/199.
} 
This paper carries out the corresponding analysis of BMS (beef marbling standards) images in China and America. Specific analysis indicators are the box-counting dimension[3], differential box-counting dimension, information dimension. Firstly, the background[4] of beef eye muscle image is removed through using the knowledge of machine vision, then extracting the longissimus dorsi. The fractal dimension of longissimus muscle is increased with the improvement of the marbling quality. After acquiring dimension of different grades marbling, regression mathematical model is established to distinguish the grade.

\section{$1 \quad$ Image Processing}

To get rid of some interference information, background of the beef image should be removed .Then, by the method of binarization, mathematical morphology, sent shadow method, etc, the fat and proud flesh which surround beef eye muscle[5] can be removed. Sometimes part of the proud flesh and longissimus muscle contact too close to be divided, because artificial boundaries can open them, so achieving the ultimate goal would be no problem.

\subsection{Background Removed}

Background removal is benefit to extract the longissimus muscle[6]. Threshold method is used. The background is black, while the beef is red and white color, so the difference is significant. Standards images are only two major categories of the target and background. So only to select a threshold, it is called single threshold [7] split. This approach let the gray value of each pixel in the image to compare with threshold. The gray value of pixels which are greater than the threshold is for a class, and the gray value of pixels which are less than the threshold value is to the other class. Significant color is different [8] at the background and beef, so selecting a appropriate threshold[9], the background can be removed.

Fig. 1 are the third grade image of USA, and the effective image of background removal. To make the effect more obvious, the background is set to white.

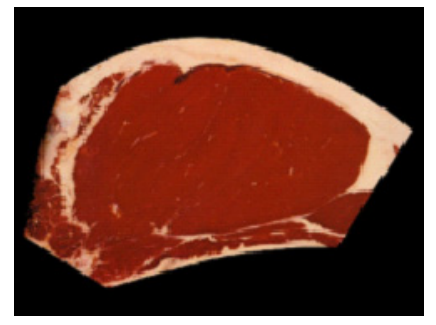

(a). The level 3 figure

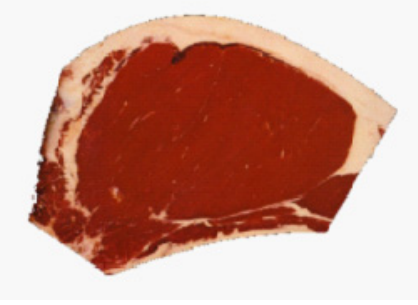

(b). Background eliminate

Fig. 1. Third grade image of America removes background 


\subsection{The Extraction of Longest Back Muscle}

The peripheral of beef eye muscle is the fat and proud flesh[10].To extract the longissimus dorsi, they should be get rid. The image type conversion, mathematical morphology[11], the mark function, properties function, sent shadow method, image restoration, etc ,are used [12] to get the longissimus muscle.

Fig. 2 show the American seventh level image and processed result.

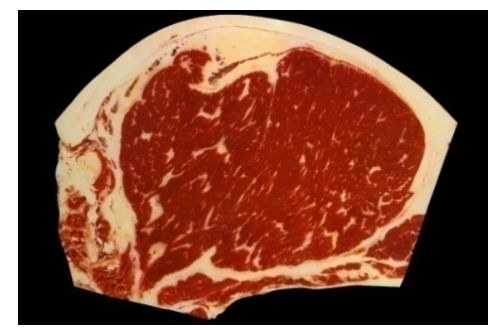

(c). American 7th level image

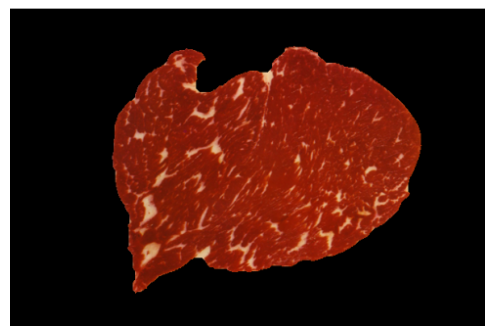

(d). Longissimus muscle

Fig. 2. The American 7th level image and processed result

For some images, some proud flesh and longissimus muscle is too tight[13] to be separated. For example, Fig. 3 show third level image and processed result. This requires other methods, so several methods are used to compare for the best.
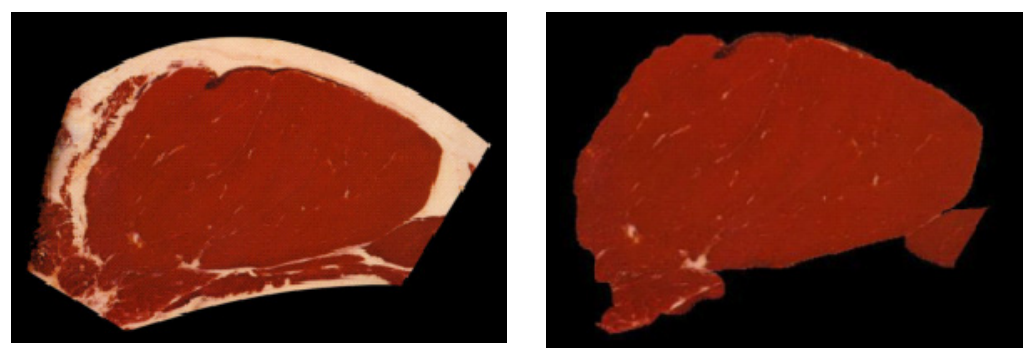

(e). Level 3 original

(f). Longissimus muscle diagram with proud flesh

Fig. 3. The third level image and processed result

The shape of longissimus muscle likes an oval. Tring to draw a white oval with right size to be the artificial boundaries, the effect is well as shown in Fig.4-(h). 


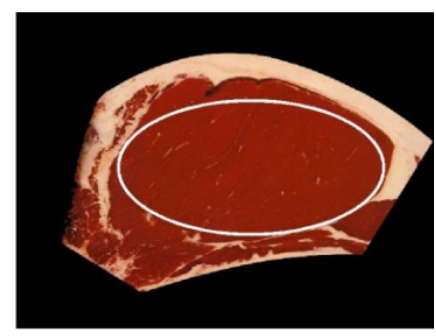

(g).To add a ellipse

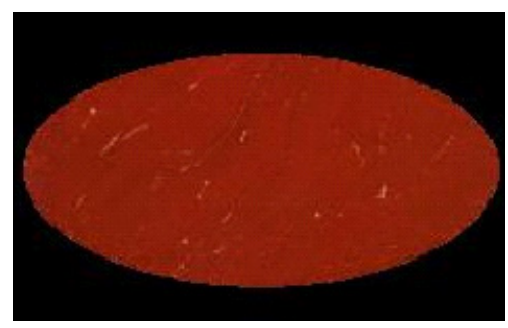

(h). Processed results

Fig. 4. Adding ellipse and then extracting the longissimus dorsi muscle

Fig.4- (h) has no proud flesh, but longissimus muscle area decreases.

The iterative morphology method is also used. Firstly, let Fig.3-(f) be binary. In addition , structural element of "disk" [14] is used. Figure erodes and dilates four times, and then using a "for" loop program[15] which makes the image to recruit into RGB diagram.

Fig.5 shows the effect. However, Fig.5 still has a few proud flesh which is not removed, and a few part of longissimus muscle is split off. It is better than Fig.4(h).Although, the best method is needed.

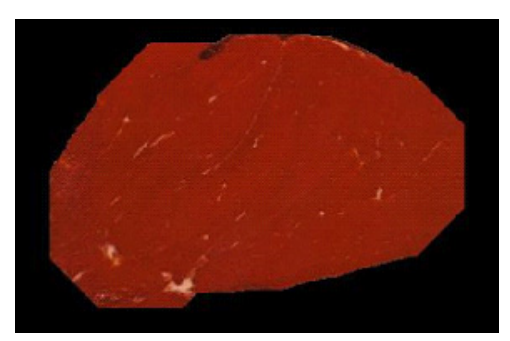

Fig. 5. Iterative corrosion and expansion to image

Fig.4-(g) paints oval to remove proud flesh, but longissimus muscle is lost a few. To further improve the method, if at the close connection of proud flesh and longissimus muscle, drawing a curve to be artificial boundaries, the effect may be well.

On the third level image of the United States, at the closely connection of proud flesh and longissimus dorsi, two white curves are drawn. Then dealing with separately [16], the proud flesh is removed, as shown in Fig.6. 


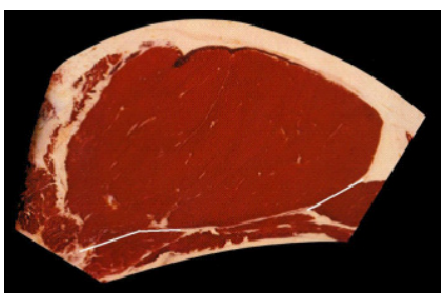

(i) .Add curves

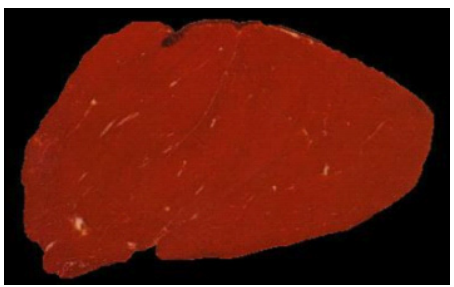

(j). Processing results

Fig. 6. Drawing curves and then extracting the longissimus muscle

Some standards images, at the departments of proud flesh and longissimus muscle connecting closely, also do this processing, the result is quite good too. Fig.7 are longissimus muscle diagrams of Chinese second and third grade images,for example.

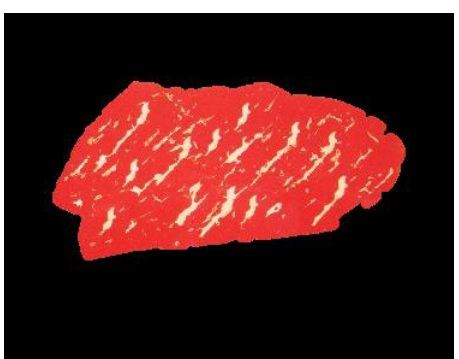

(k). Longissimus muscle of Level 2

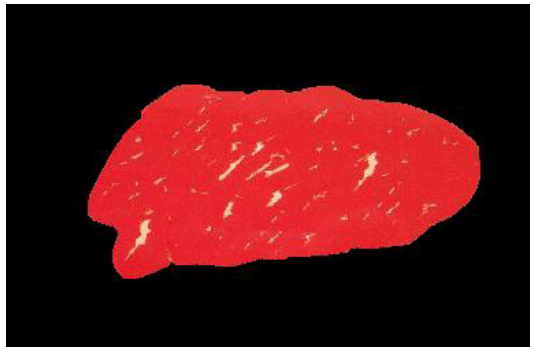

(1). Longissimus muscle of Level 3

Fig. 7. Longissimus muscle diagrams of Chinese standards images.

\section{The Application of Fractal Dimension}

Four kinds of fractal dimension calculation methods and MATLAB software[17] are used. Method 1 is traditional box-counting dimension algorithm. Method 2 is the differential box-counting algorithm. Method 3 is information dimensional algorithm. Method 4 is improved box-counting dimension algorithm. These four methods are with a number of step $\mathrm{r}_{\mathrm{i}}$ to divide the grids that cover the longissimus muscle image. If fat particles are in the mesh[18], counting them and summing to get $\mathrm{Nr}$, then marking point $\left(\log \left(1 / \mathrm{r}_{\mathrm{i}}\right), \log \left(\mathrm{Nr}_{\mathrm{i}}\right)\right)$ on a double logarithmic coordinates, fitting them with a straight line, finally absolute value of the line's slope is dimension value.

\subsection{Compare Fractal Dimension Calculation Method}

\subsubsection{Method One Is Traditional Box-Counting Dimension Algorithm}

The calculation of the box-counting dimension, intuitive understanding, is counting the number of lattice [19]. Binary image is covered with small square box of different side length, and different side length of the small square box to cover it, the box number is also different. Side length $r$ and a total of not empty small box N(r), meet 
the relationship test: $D_{b}=-\lg N(r) / \lg (1 / r)$. $D_{b}$ is the box dimension. Scale size $r$ is usually $2^{\wedge} \mathrm{n}$. A series of non-empty box number are acquired in the different proportion sizes of $r$. The reciprocal of $r$, and the number of these non-empty boxes, are set in double logarithmic coordinate[20], by least squares linear to fit them, then the absolute value of slope is the box dimension. Table 1 is results of this method to obtain.

Table 1. Results of traditional box counting dimension method for calculating values

\begin{tabular}{ccccccc}
\hline American level & Level 3 & Level 4 & Level 5 & Level 6 & Level 7 & Level 8 \\
Fractal dimension & 1.2222 & 1.2324 & 1.2343 & 1.2185 & 1.2314 & 1.2204 \\
\hline Chinese level & Level 1 & Level 2 & Level 3 & Level 4 & & \\
Fractal dimension & 1.2719 & 1.2763 & 1.2714 & 1.2751 & & \\
\hline
\end{tabular}

This method is time-consuming[21] a bit long, about three seconds. The law of increasing dimension value with the high quality of marbling is less obvious.

\subsubsection{Method Two Is Differential Box-Counting Dimension Algorithm}

Differential box-counting algorithm[22] has three dimensions. The third dimension is the image gray. Let $M \times M$ size image divided into $S \times S$ sub-block $(M / 2 \geq S>1, S$ for integer), and $r=S / M$. To imagine the images into surfaces[23] of threedimensional space, $\mathrm{x}, \mathrm{y}$ represents plane position, and $\mathrm{z}$-axis represents the gray value. $\mathrm{X}-\mathrm{Y}$ plane is divided into a lot of $\mathrm{s} \times \mathrm{s}$ grid. In each grid is an $\mathrm{s} \times \mathrm{s} \times \mathrm{s}$ box. For different $r$ and the calculation of non-empty box $\mathrm{N}_{\mathrm{r}}$, using the least squares linear to fit, the fractal dimension $\mathrm{D}$ could be obtained. The calculation results are shown in Table 2 .

Table 2. Results of differential box-counting dimension method to calculate values

\begin{tabular}{ccccccc}
\hline American level & Level 3 & Level 4 & Level 5 & Level 6 & Level 7 & Level 8 \\
Fractal dimension & 2.1263 & 2.1253 & 2.1450 & 2.1250 & 2.1352 & 2.1322 \\
\hline Chinese level & Level 1 & Level 2 & Level 3 & Level 4 & & \\
Fractal dimension & 2.2983 & 2.2485 & 2.1709 & 2.1139 & & \\
\hline
\end{tabular}

The differential box-counting algorithm, to the Chinese images, the values are well; but the images to the United States, less than ideal, the law that the fractal dimension value increments with high quality of marbling is not very obvious.

\subsubsection{The Third Method Is the Information Dimension}

This method is similar to traditional box-counting dimension algorithm[24]. Let $\mathrm{N}$ be the total fat information elements, $\mathrm{N}_{\mathrm{i}}$ is fat information on the number of elements contained in each cover, the probability that fat distribution of information in each 
coverage is: $P_{i}=N_{i} / N$. Amount of fat information is $I_{i}=-P_{i} \ln P_{i}$. The amount of fat information is I (r). Changing the scale $\mathrm{r}$, I (r) with the $1 / \mathrm{r}$ meet the relationship test: $\mathrm{I}(\mathrm{r}) \propto(1 / \mathrm{r}) \mathrm{D}$. D is its information dimension. Results are shown in Table 3.

Table 3. Results of information dimension method to calculate values

\begin{tabular}{crrrrrr}
\hline American level & Level 3 & Level 4 & Level 5 & Level 6 & Level 7 & Level 8 \\
Fractal dimension & 1.7516 & 1.7479 & 1.7492 & 1.7500 & 1.7521 & 1.7624 \\
\hline Chinese level & Level 1 & Level 2 & Level 3 & Level 4 & & \\
Fractal dimension & 1.6978 & 1.6834 & 1.6667 & 1.6942 & & \\
\hline
\end{tabular}

Information dimension method, its effect is not very satisfactory. The law ${ }^{[25]}$ that incremental dimension value with the high quality of marbling is also not very obvious.

\subsubsection{Method Four Is an Improved Box-Counting Dimension}

For the above three methods, the values which are obtained are not very satisfactory. Method four is proposed: an improved box-counting dimension. It is used in the binary image of the longissimus dorsi. Method four has improvements and advantages as follows.

2.1.4.1 Square (box) side lengths are with a lot of data, meaning that the mesh number of times is a lot, not just the $2^{\wedge} \mathrm{n}$. It can help improve data accuracy.

2.1.4.2 When the image size could not be a square side length divisible, then rounding the excess with only small "margins" section, thus reducing outside interference.

2.1.4.3 It gives value "1" pixel of binary chart, accounting for the entire map of the area proportion. This reflects objectively the number of marble pattern.

2.1.4.4 D numerical is strong regularity that they show an increasing trend with marbling levels increase.

2.1.4.5 Calculation of the dimension values is short time-consuming. Evaluating the code, the results show out immediately.

The calculation results of method four are shown in Table 4.

Table 4. Results of improved box-counting dimension method to calculate values

\begin{tabular}{ccccccc}
\hline American level & Level 3 & Level 4 & Level 5 & Level 6 & Level 7 & Level 8 \\
Fractal dimension & 1.8159 & 1.8242 & 1.8575 & 1.8646 & 1.8754 & 1.8960 \\
\hline Chinese level & Level 1 & Level 2 & Level 3 & Level 4 & & \\
Fractal dimension & 1.9630 & 1.9521 & 1.9168 & 1.8819 & & \\
\hline
\end{tabular}


This method is time-consuming short. When the program is evaluated, the results show out immediately. In the images of China and the United States, the values obtained increase with the quality of marbling became high.

After comprehensive comparison, method 4, an improved box-counting dimension method is the best, so use it to judge the grade.

\subsection{Calculating and Testing of Improved Box-Counting Dimension Method}

Detailed results of calculation and test are in Table 5 and Table 6. Exel is used to test significance of calculated data through method 4 . That is to view $\mathrm{R}^{2}$ value of fitting equation.

Table 5. Fitting results of American images' fractal dimension value

\begin{tabular}{cccc}
\hline American level & Fitting equation & Dimension & $\mathrm{R}^{2}$ \\
\hline 3 & $\mathrm{y}=-1.8159 \mathrm{x}+11.9629$ & 1.8159 & 0.9894 \\
4 & $\mathrm{y}=-1.8242 \mathrm{x}+11.9983$ & 1.8242 & 0.9899 \\
5 & $\mathrm{y}=-1.8578 \mathrm{x}+12.1418$ & 1.8578 & 0.9914 \\
6 & $\mathrm{y}=-1.8646 \mathrm{x}+12.1702$ & 1.8646 & 0.9919 \\
7 & $\mathrm{y}=-1.8753 \mathrm{x}+12.2148$ & 1.8753 & 0.9925 \\
8 & $\mathrm{y}=-1.8960 \mathrm{x}+12.3010$ & 1.8960 & 0.9936 \\
\hline
\end{tabular}

Table 6. Fitting results of Chinese images' fractal dimension value

\begin{tabular}{ccrc}
\hline Chinese level & Fitting equation & Dimension & $\mathrm{R}^{2}$ \\
\hline 1 & $\mathrm{y}=-1.9630 \mathrm{x}+11.8332$ & 1.9630 & 0.9936 \\
2 & $\mathrm{y}=-1.9521 \mathrm{x}+11.7887$ & 1.9521 & 0.9927 \\
3 & $\mathrm{y}=-1.9168 \mathrm{x}+11.6423$ & 1.9168 & 0.9906 \\
4 & $\mathrm{y}=-1.8947 \mathrm{x}+11.5479$ & 1.8947 & 0.9899 \\
\hline
\end{tabular}

Seeing from the tables, the fitting equations are obvious in significance level.

Two images'fitting maps are giving out. Fig.8 are the fitting diagram of the seventh grade of USA, and third grade of China.

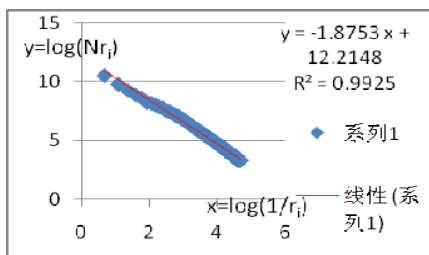

(m). Fitting figure of American seventh grade image

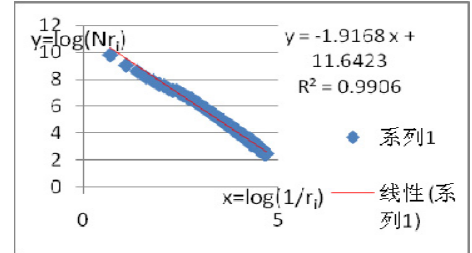

(n). Fitting figure of Chinese third grade image

Fig. 8. Two standards images fit in diagram 


\section{Marbling Grade of Mathematical Model}

\subsection{Mathematical Model of American Images}

Assuming that the regression equation is: $\mathrm{T}=\mathrm{a}+\mathrm{b} * \mathrm{D}$.Level value $\mathrm{T}$ with the boxcounting dimension value $\mathrm{D}$, as well as the $\mathrm{T}^{2}, \mathrm{D}^{2}, \mathrm{D} * \mathrm{~T}$, etc ${ }^{[26]}$, are used. Through regression analysis, a regression equation is established about the American images:

$$
\mathrm{T}=-105.9740+60.0746 \mathrm{D}
$$

$\mathrm{T}$ is level value, and $\mathrm{D}$ is fractal dimension value in equation (1).Through F-test, the equation is significant at level of $\alpha=0.05$.

\subsection{Test of Equation (1)}

Results of the equation (1) are examined, only validation of level 5 is mistaken. The accuracy rate is $83.33 \%$, as shown in Table 7 .

Table 7. Level verification of equation (1)

\begin{tabular}{ccc}
\hline The actual level & Calculated value & Round \\
\hline 3 & 3.1155 & 3 \\
4 & 3.6141 & 4 \\
5 & 5.6146 & 6 \\
6 & 6.0411 & 6 \\
7 & 6.6899 & 7 \\
8 & 7.9274 & 8 \\
\hline
\end{tabular}

\subsection{Mathematical Model of Chinese Images}

Similarly, through regression analysis, combined with the corresponding value, regression equation is established:

$$
\mathrm{T}=68.7368-34.3471 \mathrm{D}
$$

$\mathrm{T}$ is level value, and $\mathrm{D}$ is fractal dimension value in equation (2).Through F-test, the equation is significant at level of $\alpha=0.05$.

\subsection{Test of Equation (2)}

Results of the equation (2) are examined.The accuracy rate is $100 \%$, as shown in Table 8 . 
Table 8. Level verification of equation (2)

\begin{tabular}{ccc}
\hline The actual level & Calculated value & Round \\
\hline 1 & 1.3134 & 1 \\
2 & 1.6876 & 2 \\
3 & 2.9003 & 3 \\
4 & 4.0990 & 4 \\
\hline
\end{tabular}

\section{Conclusion and Suggestion}

This study, first of all is the standards images' processing that to extraction longissimus muscle. To figures of some proud flesh and longissimus dorsi closely connected, three image segmentation methods are compared. The optimal method is to add curve manually.

For Chinese and American images, four fractal dimension methods are used. After comparison, the final choice is improved box-counting dimension algorithm. Dimensions obtained through this method are regularity, that the $\mathrm{D}$ values become larger with increased beef marbling levels.

Using regression equations to establish a linear mathematical model, the effect is well. Mathematical model of American images, the accuracy rate is $83.33 \%$; to Chinese images, the accuracy rate is $100 \%$.

BP network, and support vector machine modeling methods can be tried to compare the classification results. Some rounded values, although are correct, but they are close to the median. Trying other mathematical models, the accuracy may be higher.

\section{References}

1. National beef grading methods and standards-NY / T. 676-2010

2. Chu, D., Wang, X.: Fractal geometry in applied geophysics. Foreign Oil and Gas Technology 4(9), 44-52 (1995)

3. Chen, K.: Beef marbling box-counting dimension and information dimension determination. Agricultural Engineering 23(7), 145-149 (2007)

4. Brosnan, T., Sun, D.: Inspection and grading of agricultural and food products by computer vision systems to review. Computers and Electronics in Agriculture 36, 193-213 (2002)

5. Chen, K., Qin, C., Ji, C.: The research of cattle carcass eye flesh image segmentation method. Agricultural Machinery 37(6), 155-158 (2006)

6. Gerrard, D.E., Gao, X., Tan, J.: Beef marbling and color score determination by processing. Journal of Food Science 61(1), 145-148 (1996)

7. Chen, K., Qin, C., Mcdonald.: Segmentation of Beef Marbling based on Vision Threshold. Computers and Electronics in Agriculture 62(2), 223-230 (2008)

8. Jackman, P., Cheng, G., Jindu.: Predition of beef eating quality from colour, marbling and wavelet texture features. Meat Science 80(4), 1273-1281 (2008) 
9. Yang, H.: Image segmentation research through threshold value method. Natural Science Newspaper 33(2), 135-137 (2006)

10. McDonaId, T.P., Chen.: Separating connected muscle issue on images of beef carcass eyes meat. Trans. of ASAE 55(6), 2059-2065 (1990)

11. Ren, F., Tu, K., et al.: Application of image processing technology evaluation of beef marbling. Meat Research (4), 14-15 (2002)

12. Shiraniata, K., Hayashi, K., et al.: Grading meat quality by image processing. Pattern Recognition 33, 97-104 (2000)

13. Zhao, J., Liu, M., Zhang, H.: Research of beef longissimus dorsi image split and marbling extracting technique based on mathematical morphology. Agricultural Engineering 20(1), 144-146 (2004)

14. Hu, X., Dong, C.: MATLAB from entry to master. People's Posts and Telecommunications Press (2010)

15. Shen, Z., Gao, F., Li, C., et al.: Beef grading technology based on computer vision research progress. Food Science and Technology 29(6), 304-306 (2008)

16. Quevedo, R., Calos, L.G., Aguilera, J.M., Cadoche, L.: Description of food surfaces and microstructural changes using fractal image texture analysis. Journal of Food Engineering 53(4), 361-371 (2002)

17. Tan, J.L.: Meat quality evaluation by computer vision. Journal of Food Engineering 61, 27-35 (2004)

18. Sun, Y., Xianyu, J., Shi, J.: The computer vision-based analysis method of cooling beef tenderness. Agricultural Machinery 34(5), 102-105 (2003)

19. Li, P., Xing, L., Pan, J., Gu, X.: Calculation of the fractal dimension and the image edge extraction. Journal of Jilin University (Information Science) 29(2), 152-156 (2011)

20. Pentland, P.: Fractal-based description of natural scenes. IEEE Transactions on Pattern analysis and Machine Intelligence 6(6), 661-674 (1984)

21. Zhang, Q., Wang, Z.: Proficient in MATLAB image processing. Electronics Industry Publishing House (2009)

22. Zhang, J.: Fractal, 2nd edn. singhua University Press (2011)

23. Liu, M., Zhang, X.: Differential box dimension method on the weld edge detection. Hebei Industrial Technology 26(5), 300-302 (2009)

24. Yin, Y., Li, P., Kang, Y., et al.: A Survey and Research of fractal theory. Science and Technology (15), 152-170 (2007)

25. Zhao, H., Yang, G., Xu, Z.: The comparing of Image fractal dimension calculation methods. Computer System Apply 20(3), 238-241, 246 (2010)

26. Li, Y., Hu, C.: Experimental design and data processing, 2nd edn., pp. 82-93. Beijing Chemical Industry Press (2011) 